\title{
Productive potential and castor bean selection of the FCA-PB cultivar progenies ${ }^{1}$
}

\author{
Jackson da Silva', Andréia Rodrigues Ramos ${ }^{3}$, Deoclécio Jardim Amorim, \\ Mauricio Dutra Zanotto ${ }^{3}$, Maria Márcia Pereira Sartori ${ }^{3 *}$ (D)
}

10.1590/0034-737X202067010007

\begin{abstract}
Castor bean is a very important crop, however, in Brazil it still has relatively low productivity, $0.47 \mathrm{Mg} \mathrm{ha}^{-1}$ as a mean. Besides that the Country has significant castor oil demand and depends on international commerce to complement its production. The solution of these problems goes through the development of genotypes highly productive and adapted to the producing regions. So, the objective of this research was to evaluate the productive potential and selection of castor progenies of the cultivar FCA-PB, resulting from three types of pollination, in two crops and in two localities of the State of São Paulo. Grain productivity was evaluated, and some genetic parameters were estimated. In relation to the 2005/2006 crop, the progeny 49 cultivated in the locality of São Manuel produced $4170.66 \mathrm{~kg}$ ha $^{-1}$ of grains. The highest heritability coefficients were observed in the 2005/2006 crop in the locacity of Araçatuba. Progenies 1, 5, 6, 8, 15, 18, 19, 21, 27, 31, 35, 36, 38, 45, 49, 55, 56 and 58 were selected. Genotype interaction with the location indicated productive materials and adapted to the state of São Paulo.
\end{abstract}

Keywords: Ricinus communis L.; breeding; pure lines; stability.

\section{INTRODUCTION}

The castor bean belongs to the family Euphorbiacea, genus Ricinus and species Ricinus communis L. according to Ferreira (2006), this culture belongs to the center of origin Absinthia, classified by Vavilov, being this region the place that one finds the greater genetic diversity of the species. This crop has many applications in the industrial area and in the production of biodiesel, having thus, relevant economic importance (Goneli et al., 2018).

Brazil is the fourth largest producer in the world, with production of 24 thousand tons and relatively low productivity with $0.47 \mathrm{Mg} \mathrm{ha}^{-1}$, being the national production concentrated in the Northeast with $88.54 \%$, where the State of Bahia is the country's largest producer with a production of 10.4 thousand tons, presenting productivity of $0.49 \mathrm{Mg} \mathrm{ha}^{-1}$ (FAO, 2016; CONAB, 2017).
Although the country is one of the world's leading producers of this crop, according to FAO (2016), this year the Brazil imported 3621 tons to meet the industry demand this oil. Thus, there is a demand for the production of castor oil, which can be achieved with the development of highly productive genotypes besides being adapted to the edaphoclimatic conditions of each producing region (Daronch et al., 2019).

The development of these genotypes depends on genetic improvement, in which one can glimpse the obtaining of varieties, hybrids and pure lines, being the pollination a factor that reflects in the homozygous level and, consequently, on the purpose of the genotype. In addition, genetic improvement still involves the study of the adaptation of a given genotype to a certain location, hence the importance of evaluating in different years and

\footnotetext{
Submitted on March 1st, 2019 and accepted on November $1^{s t}, 2019$.

I This work is part of the master's thesis of first author.

${ }^{2}$ Universidade Federal Rural de Pernambuco, Recife, Pernambuco, Brazil. jackson.silva.batalha@gmail.com

${ }^{3}$ Universidade Estadual Paulista “Júlio de Mesquita Filho”, Botucatu, São Paulo, Brazil. andreia-agro@hotmail.com; deocleciojardim@hotmail.com; zanotto@fca.unesp.br; maria.mp.sartori@unesp.br

*Corresponding author: maria.mp.sartori@unesp.br
} 
places, because this increases the accuracy of the breeder when selecting the best genotypes (Cruz et al., 2012).

Selection of superior genotypes can be hampered because the characteristics are controlled by several genes, in particular the quantitative characteristics, such as grain yield. However, the use of genetic parameters, such as heritability and genetic gain, can guide the selection, in order to make it more efficient (Gaya et al., 2006).

In view of the foregoing, the objective of the present research was to evaluate the productive potential and castor bean selection of the FCA-PB cultivar progenies, resulting from three types of pollination, in two crops and in two localities of the State of São Paulo.

\section{MATERIALAND METHODS}

\section{Location and characterization of experimental areas}

The experiments were developed in the 2004/2005 and 2005/2006 crop seasons, in the months of October to May, simultaneously in two localities of the State of São Paulo, São Manuel and Araçatuba. The climate of the city of São Manuel-SP, is of type Cfa, the average annual temperature is between 18 to $20^{\circ} \mathrm{C}$, with annual average rainfall between 1000 and $1300 \mathrm{~mm}$ and altitude between 600 and 800 meters. In relation to the city Araçatuba, according to the classification of Köppen-Geiger is type Aw, presenting an average annual temperature of $22.2^{\circ} \mathrm{C}$, with annual average rainfall of $1206 \mathrm{~mm}$ and altitude around 401 meters (Alvares et al., 2014).

\section{Experimental design and treatments}

The experiments were in design in randomized blocks, in the factorial scheme $90 \times 2 \times 2$, being 90 progenies (coming from three types of pollination, free pollination (progenies 01 to 30 ), cross-pollination (progenies 31 to 60 ) and selffertilization (progenies 61 to 90)), 2 localities (São Manuel and Araçatuba) and 2 agricultural crops (2004/2005 and 2005/2006), with three replications. It is noteworthy that castor bean progenies were obtained from the FCA-PB population (developed by Program for the Improvement of castor beans of UNESP/FCA).

\section{Installation and conduction of experiments}

Prior to the installation of the experiment, soil samples were drawn at a depth of 0.0 to $0.2 \mathrm{~m}$. With the results, was carried out the fertilization of foundation with $400 \mathrm{~kg} \mathrm{ha}^{-1}$ of formulated fertilizer 04-14-08.

Soil preparation was carried out in a traditional way with two plows and two harrows. Subsequently, the grooves were opened and the fertilizer was distributed with a seeder-fertilizer tratorized, adjusted according to the spacing of $1.0 \mathrm{~m}$ between rows.
Plantings of the experiments occurred in October 2004 and 2005, where three seeds were manually placed every $0.5 \mathrm{~m}$ of furrows, with depths of 4 to 8 centimeters, and ten days after the emergency the thinning was left leaving two plants per linear meter.

The experimental plot consisted of 3 lines of $7 \mathrm{~m}$ in length, having used the spacing of $1.0 \times 0.5 \mathrm{~m}$, where they were considered as useful area, for data collection, the central row, discarding the first two plants of each end.

To ensure the germination and uniformity of the plants, so that the maintenance of soil moisture for the crop occurred ideally, a sprinkler irrigation system was installed, with a blade varying according to the evapotranspiration of castor bean, this system was only used in the initial phase of the culture.

Due to the long cycle of this culture, weed control was carried out at three times during the crop cycle, being this control occurred manually.

\section{Evaluated characters}

The crops were harvested in May 2005 and 2006, in which the evaluation was given of grain yield (PG), in kg $\mathrm{ha}^{-1}$, having weighed the seeds of the five central plants of the plot, corrected to $13 \%$ moisture and, in followed estimated for hectare.

\section{Statistical analysis}

The measured data were submitted to analysis of variance and joint analysis of variance, considering the effects of treatments as fixed. And when necessary, the comparison was applied between the means of the treatments by the Scott-Knott test, at the $5 \%$ probability level, with the use of AgroEstat software (Barbosa \& Maldonado Junior, 2015).

\section{Determination of genetic parameters}

With the productivity data in hand, were calculated some genetic parameters: heritability to the average level, expected genetic gain and the estimated genetic progress in percentage, as detailed in equations 1,2, 3 and 4, reported by Cruz et al. (2012), being the equations expressed as follows:

$h^{2} m=\frac{\mathrm{QMP}-\mathrm{QME}}{\mathrm{QMP}}$

In what: $h^{2} \mathrm{~m}=$ heritability at the level of progeny average $\mathrm{QMP}=$ Average square of progeny

$\mathrm{QME}=$ Average square of the residue

$\Delta G=i \frac{\sigma^{2} p}{\sqrt{\sigma^{2} p+\frac{\sigma^{2} e}{r}}}$ 
In which: $\Delta \mathrm{G}=$ Estimate of expected genetic progress with $20 \%$ of selection intensity in $\mathrm{kg} \mathrm{ha}^{-1}$.

$\mathrm{i}=1.4$ standardized selection differential, corresponding to $20 \%$ of selection intensity (Vencovsky, 1980).

$\sigma^{2} \mathrm{p}=$ Genetic variance between progenies $\left(\mathrm{kg} \mathrm{ha}^{-1}\right)^{2}$, obtained by

$\sigma^{2} \mathrm{p}=\frac{\mathrm{QML}-\mathrm{QME}}{r}$

$\mathrm{r}=$ Number of repetitions.

$\sigma^{2} \mathrm{e}=\mathrm{QME}$

$\mathrm{G} \%=\frac{\Delta \mathrm{G}}{\widehat{\mathrm{m}}} \cdot 100$

In which: $\mathrm{G} \%=$ Genetic progress estimated in percentage in relation to the average of productivity of each progeny.

$\Delta \mathrm{G}=$ Estimated expected genetic progress with $20 \%$ of selection intensity in $\mathrm{kg} \mathrm{ha}^{-1}$.

$\hat{\mathrm{m}}=$ Average productivity of each progeny.

\section{RESULTS AND DISCUSSION}

It is observed by the analysis of joint variance between progenies, localities and crops (Table 1) that the interaction between and harvests showed no significant behavior at $5 \%$ probability by the $\mathrm{F}$ test, indicating that there was no influence between locality and crop, suggesting that the progeny group, on average, shows similar behavior for grain in the two localities and two crops.

Table 1: Analysis of joint variance between progenies, localities and crops in relation the characteristic grain yield $\left(\mathrm{kg} \mathrm{ha}^{-1}\right)$

\begin{tabular}{lcc}
\hline Source of variation & $\mathbf{G L}^{(\mathbf{1})}$ & $\mathbf{Q M}^{(2)}$ \\
\hline Block / Location & 4 & 2444572.63 \\
Location & 1 & $6191957.57^{* *}$ \\
Crop & 1 & $2011912.03^{* *}$ \\
Progeny & 89 & $1383597.44^{* *}$ \\
$\quad$ Free pollination & 29 & $1517654.72^{* *}$ \\
$\quad$ Cross pollination & 29 & $937153.98^{* *}$ \\
$\quad$ Self-pollination & 29 & $1361299.33^{* *}$ \\
$\quad$ Between Progeny & 2 & $6236519.81^{* *}$ \\
Location x Crop & 1 & $159870.00 \mathrm{~ns}$ \\
Location x Progeny & 89 & $1093284.65^{* *}$ \\
Crop x Progeny & 89 & $918884.95^{* *}$ \\
Location x Crop x Progeny & 89 & $1132049.00^{* *}$ \\
\hline Treatment & 359 & \\
\hline Residue & 716 & 119344.65 \\
Total & 1079 & \\
\hline CV & \\
\hline ** Significant at the 1\% probability level and ns not significant at \\
5\% probability, both by test F. 1: Degrees of freedom; 2: Average \\
square; 3: Coefficient of variation.
\end{tabular}

However, in all other sources of variation were detected the respective significance at the $1 \%$ probability level by the $\mathrm{F}$ test, thus observing variation among the progenies in general, as well as between types of pollination and between the progenies in each method of pollination, indicating significant gains with the selection of the best progenies.

Due to the significance of the interaction progeny $\mathrm{x}$ crop $x$ location at the $1 \%$ probability level by the $\mathrm{F}$ test, the analysis of joint variance was broken down into two, being a joint analysis of variance, progenies and local and the other progenies and crops.

In the analysis of joint variance between progenies and local for each crop (Table 2), It is observed that the progenies and the localities obtained the same behavior in the two crops, taking the local and progeny factors, in general, presented significant non-significant effects at the $5 \%$ probability level and the interaction progeny $\mathrm{x}$ location, free pollination, cross-pollination, self-pollination and between these three types of pollination significance at the $1 \%$ probability level by the $\mathrm{F}$ test.

Due to the significance of the analysis of joint variance, it was necessary to apply the comparison test between averages (Table 3), it can be observed that, in crop 2004/ $2005,54 \%$ of the progenies presented a difference in productivity between the localities, and in the 2005/2006 crop $44 \%$.

As the progeny $\mathrm{x}$ local interaction was significant and most of the progenies present differences between the production localities it is natural and important in genetic breeding that each progeny best suit a given environment, because in this way the breeders can act to select genotypes with a higher level of phenotypic stability, as well as the development of cultivars or lines adapted to a specific region, at the expense of genetic gains and precision in selection (Torres et al., 2015).

Thus, the progenies that presented the highest productivity of grains in the 2004/2005 crop in São Manuel were: $1,3,5,7,10,22,31,33,35,37,38,40,52,78,82,86$ and 89 , with a variation of 2729 to $3262 \mathrm{~kg} \mathrm{ha}^{-1}$ and in Araçatuba $8,28,30,32,42,43,44,46,49,55,56,57,61$ and 68 , being the variation of 3107 to $3904 \mathrm{~kg} \mathrm{ha}^{-1}$. Indicating the genetic potential of the aforementioned progenies, and detaining yields about five times higher in comparison to the national productivity in the crop of the experiments (CONAB, 2017).

In relation to the 2005/2006 crop, the progeny 49 grown in the locality of São Manuel reached $4171 \mathrm{~kg} \mathrm{ha}^{-1}$ of grain yield and, the progeny 81 in the city of Araçatuba obtained yield of $4453 \mathrm{~kg} \mathrm{ha}^{-1}$. In that the high productivity of the progeny 49 can be explained by the type of fertilization that originated, which was by cross-pollination, being this method that causes the highest level of heterosis, and this progeny can result in highly productive hybrids, as it is 
Table 2: Analysis of joint variance between progenies and localities, for each crop, in relation to the characteristic grain yield $\left(\mathrm{kg} \mathrm{ha}^{-1}\right)$

\begin{tabular}{lccc}
\hline \multirow{2}{*}{ Source of variation } & GL $^{(\mathbf{1})}$ & \multicolumn{2}{c}{$\mathbf{Q M}^{(2)}$} \\
\cline { 3 - 4 } & & Crop 2004/2005 & Crop 2005/2006 \\
\hline Block / Location & 4 & 1515510.49 & 1141257.40 \\
Location & 1 & $4170855.12 \mathrm{~ns}$ & $2180972.45 \mathrm{~ns}$ \\
Progeny & 89 & $1042230.68 \mathrm{~ns}$ & $1260251.71 \mathrm{~ns}$ \\
$\quad$ Free pollination & 29 & $1235607.81 * *$ & $986907.17 * *$ \\
$\quad$ Cross pollination & 29 & $647885.86 * *$ & $929027.44 * *$ \\
$\quad$ Self-pollination & 29 & $827185.07 * *$ & $1360333.61 * *$ \\
$\quad$ Between Progeny & 2 & $7074423.67 * *$ & $8575311.77 * *$ \\
Progeny x Location & 89 & $1235473.31 * *$ & $989860.34 * *$ \\
\hline Treatment & 179 & 114169.77 \\
\hline Residue & 356 & 123476.26 & 14.66 \\
Total & 539 & 15.87 & \\
\hline CV & $(3)$ & & \\
$* *$ and ns: Significant at the 1\% probability level and not significant at 5\% probability, respectively, both by the test F. 1: Degrees of
\end{tabular}

Table 3: Averages of grain yield $\left(\mathrm{kg} \mathrm{ha}^{-1}\right)$ of progenies and localities, for each crop

\begin{tabular}{|c|c|c|c|c|c|}
\hline \multirow{2}{*}{ Progeny } & \multicolumn{2}{|c|}{ Crop 2004/2005 } & \multirow{2}{*}{ Progeny } & \multicolumn{2}{|c|}{ Crop 2005/2006 } \\
\hline & São Manuel & Araçatuba & & São Manuel & Araçatuba \\
\hline 1 & $2887 \mathrm{aA}^{(1)}$ & 2494 bA & 1 & 2946 bA & $2686 \mathrm{dA}$ \\
\hline 2 & $1867 \mathrm{cA}$ & $1463 \mathrm{dA}$ & 2 & $1860 \mathrm{dA}$ & $2412 \mathrm{dA}$ \\
\hline 3 & $2821 \mathrm{aA}$ & $1772 \mathrm{~dB}$ & 3 & $2772 \mathrm{cA}$ & $2662 \mathrm{dA}$ \\
\hline 4 & $1990 \mathrm{cA}$ & $1503 \mathrm{dA}$ & 4 & $2004 \mathrm{~dB}$ & $3096 \mathrm{dA}$ \\
\hline 5 & $3002 \mathrm{aA}$ & $2055 \mathrm{cB}$ & 5 & $2988 \mathrm{bA}$ & $2455 \mathrm{dA}$ \\
\hline 6 & $2240 \mathrm{bA}$ & $2805 \mathrm{bA}$ & 6 & $2208 \mathrm{dA}$ & $2171 \mathrm{eA}$ \\
\hline 7 & $2887 \mathrm{aA}$ & $1584 \mathrm{~dB}$ & 7 & 2928 bA & $2848 \mathrm{dA}$ \\
\hline 8 & $3282 \mathrm{aB}$ & $3904 \mathrm{aA}$ & 8 & 3276 bA & $2255 \mathrm{eB}$ \\
\hline 9 & $1709 \mathrm{cB}$ & $2315 \mathrm{cA}$ & 9 & $1674 \mathrm{eB}$ & $3524 \mathrm{bA}$ \\
\hline 10 & $2750 \mathrm{aA}$ & $1641 \mathrm{~dB}$ & 10 & $2796 \mathrm{cA}$ & $2881 \mathrm{dA}$ \\
\hline 11 & $2048 \mathrm{cA}$ & $2614 \mathrm{bA}$ & 11 & $2028 \mathrm{~dB}$ & $3205 \mathrm{cA}$ \\
\hline 12 & $1960 \mathrm{cA}$ & $2032 \mathrm{cA}$ & 12 & $1890 \mathrm{~dB}$ & $2738 \mathrm{dA}$ \\
\hline 13 & $1255 \mathrm{~dB}$ & $2560 \mathrm{bA}$ & 13 & $1086 \mathrm{eB}$ & $2487 \mathrm{dA}$ \\
\hline 14 & $1431 \mathrm{dA}$ & $1037 \mathrm{dA}$ & 14 & $1476 \mathrm{eB}$ & $3064 \mathrm{dA}$ \\
\hline 15 & $2368 \mathrm{bA}$ & $2870 \mathrm{bA}$ & 15 & $2364 \mathrm{cA}$ & $2535 \mathrm{dA}$ \\
\hline 16 & $1591 \mathrm{cA}$ & $3122 \mathrm{aB}$ & 16 & $1500 \mathrm{eB}$ & $2930 \mathrm{dA}$ \\
\hline 17 & $1454 \mathrm{dA}$ & $1930 \mathrm{cA}$ & 17 & $1494 \mathrm{eB}$ & $2685 \mathrm{dA}$ \\
\hline 18 & $2144 \mathrm{bA}$ & $2247 \mathrm{cA}$ & 18 & $2130 \mathrm{~dB}$ & $2982 \mathrm{dA}$ \\
\hline 19 & $2189 \mathrm{bB}$ & $2778 \mathrm{bA}$ & 19 & $2190 \mathrm{dA}$ & $2622 \mathrm{dA}$ \\
\hline 20 & $1914 \mathrm{cA}$ & $1258 \mathrm{~dB}$ & 20 & $1884 \mathrm{dA}$ & $2103 \mathrm{eA}$ \\
\hline 21 & $2513 \mathrm{bA}$ & $2741 \mathrm{bA}$ & 21 & $2484 \mathrm{cB}$ & $3280 \mathrm{cA}$ \\
\hline 22 & $2976 \mathrm{aA}$ & $1359 \mathrm{~dB}$ & 22 & 2958 bA & $1132 \mathrm{gB}$ \\
\hline 23 & $1597 \mathrm{cB}$ & $2635 \mathrm{bA}$ & 23 & $1650 \mathrm{eA}$ & $1645 \mathrm{fA}$ \\
\hline 24 & $2491 \mathrm{bA}$ & $2562 \mathrm{bA}$ & 24 & $2574 \mathrm{cA}$ & $1885 \mathrm{eB}$ \\
\hline 25 & $2228 \mathrm{bA}$ & $1762 \mathrm{dA}$ & 25 & $2136 \mathrm{dA}$ & $1506 \mathrm{fB}$ \\
\hline 26 & $2497 \mathrm{bA}$ & $1911 \mathrm{cB}$ & 26 & $2520 \mathrm{cA}$ & $2187 \mathrm{eA}$ \\
\hline 27 & $2372 \mathrm{bA}$ & $2479 \mathrm{bA}$ & 27 & $2352 \mathrm{cA}$ & $2213 \mathrm{eA}$ \\
\hline 28 & $2333 \mathrm{bB}$ & $3478 \mathrm{aA}$ & 28 & $2184 \mathrm{dA}$ & 1666 fA \\
\hline 29 & $1421 \mathrm{~dB}$ & $2123 \mathrm{cA}$ & 29 & $1524 \mathrm{eA}$ & $1536 \mathrm{fA}$ \\
\hline 30 & $1462 \mathrm{~dB}$ & 3066 aA & 30 & 1488 eA & $1440 \mathrm{fA}$ \\
\hline 31 & $2796 \mathrm{aA}$ & $2669 \mathrm{bA}$ & 31 & $2167 \mathrm{~dB}$ & $3052 \mathrm{dA}$ \\
\hline 32 & $1874 \mathrm{cB}$ & $3158 \mathrm{aA}$ & 32 & $2305 \mathrm{cB}$ & 3862 bA \\
\hline 33 & $2832 \mathrm{aA}$ & $2004 \mathrm{cB}$ & 33 & $2046 \mathrm{dA}$ & $2341 \mathrm{dA}$ \\
\hline 34 & $2006 \mathrm{cA}$ & $2080 \mathrm{cA}$ & 34 & $2125 \mathrm{~dB}$ & $2706 \mathrm{dA}$ \\
\hline
\end{tabular}


Continuação

\begin{tabular}{|c|c|c|c|c|c|}
\hline 35 & $2985 \mathrm{aA}$ & $2864 \mathrm{bA}$ & 35 & $2782 \mathrm{cA}$ & $2606 \mathrm{dA}$ \\
\hline 36 & 2214 bA & $2299 \mathrm{cA}$ & 36 & $2170 \mathrm{dA}$ & $2576 \mathrm{dA}$ \\
\hline 37 & $2967 \mathrm{aA}$ & $1894 \mathrm{cB}$ & 37 & 3102 bA & $2121 \mathrm{eB}$ \\
\hline 38 & $3262 \mathrm{aA}$ & $2390 \mathrm{bB}$ & 38 & $2459 \mathrm{cA}$ & $2578 \mathrm{dA}$ \\
\hline 39 & $1695 \mathrm{cA}$ & $1645 \mathrm{dA}$ & 39 & $2338 \mathrm{cA}$ & $2706 \mathrm{dA}$ \\
\hline 40 & $2776 \mathrm{aA}$ & $1708 \mathrm{~dB}$ & 40 & $2125 \mathrm{~dB}$ & $3693 \mathrm{bA}$ \\
\hline 41 & $2090 \mathrm{bA}$ & $2360 \mathrm{cA}$ & 41 & $2314 \mathrm{cB}$ & $3759 \mathrm{bA}$ \\
\hline 42 & $1895 \mathrm{cB}$ & $3154 \mathrm{aA}$ & 42 & $2317 \mathrm{cA}$ & $2609 \mathrm{dA}$ \\
\hline 43 & $1107 \mathrm{~dB}$ & $3300 \mathrm{aA}$ & 43 & $2638 \mathrm{cA}$ & $2575 \mathrm{dA}$ \\
\hline 44 & $1681 \mathrm{cB}$ & $3307 \mathrm{aA}$ & 44 & $2864 \mathrm{bA}$ & $2696 \mathrm{dA}$ \\
\hline 45 & 2393 bA & 2941 bA & 45 & $2481 \mathrm{cA}$ & $2532 \mathrm{dA}$ \\
\hline 46 & $1621 \mathrm{cB}$ & $3407 \mathrm{aA}$ & 46 & $2508 \mathrm{cA}$ & $2151 \mathrm{eA}$ \\
\hline 47 & $1524 \mathrm{~dB}$ & $2316 \mathrm{cA}$ & 47 & $1815 \mathrm{~dB}$ & $2667 \mathrm{dA}$ \\
\hline 48 & $2180 \mathrm{bA}$ & $2586 \mathrm{bA}$ & 48 & $2409 \mathrm{cA}$ & $1994 \mathrm{eA}$ \\
\hline 49 & 2218 bB & $3297 \mathrm{aA}$ & 49 & $4171 \mathrm{aA}$ & $2253 \mathrm{eB}$ \\
\hline 50 & $1884 \mathrm{cA}$ & $1830 \mathrm{cA}$ & 50 & $2785 \mathrm{cA}$ & $2695 \mathrm{dA}$ \\
\hline 51 & $2524 \mathrm{bA}$ & $2349 \mathrm{cA}$ & 51 & $1973 \mathrm{dA}$ & $2034 \mathrm{eA}$ \\
\hline 52 & $2967 \mathrm{aA}$ & $1544 \mathrm{~dB}$ & 52 & $1725 \mathrm{eB}$ & $2825 \mathrm{dA}$ \\
\hline 53 & $1618 \mathrm{cB}$ & $2600 \mathrm{bA}$ & 53 & $1953 \mathrm{~dB}$ & $2561 \mathrm{dA}$ \\
\hline 54 & $2521 \mathrm{bA}$ & $2485 \mathrm{bA}$ & 54 & $1973 \mathrm{dA}$ & $1517 \mathrm{fA}$ \\
\hline 55 & $2220 \mathrm{bB}$ & $3107 \mathrm{aA}$ & 55 & $3361 \mathrm{bA}$ & $2960 \mathrm{dA}$ \\
\hline 56 & 2519 bB & $3377 \mathrm{aA}$ & 56 & $2536 \mathrm{cA}$ & $2424 \mathrm{dA}$ \\
\hline 57 & $2358 \mathrm{bB}$ & $3219 \mathrm{aA}$ & 57 & $2587 \mathrm{cA}$ & $1761 \mathrm{fB}$ \\
\hline 58 & $2316 \mathrm{bA}$ & $2748 \mathrm{bA}$ & 58 & $2620 \mathrm{cB}$ & $3639 \mathrm{bA}$ \\
\hline 59 & $1443 \mathrm{~dB}$ & $2251 \mathrm{cA}$ & 59 & $2106 \mathrm{~dB}$ & $2661 \mathrm{dA}$ \\
\hline 60 & $1549 \mathrm{~dB}$ & $2793 \mathrm{bA}$ & 60 & $1536 \mathrm{eA}$ & $1677 \mathrm{fA}$ \\
\hline 61 & $1462 \mathrm{~dB}$ & $3665 \mathrm{aA}$ & 61 & $1916 \mathrm{dA}$ & $1144 \mathrm{gB}$ \\
\hline 62 & 2369 bA & $2733 \mathrm{bA}$ & 62 & $2279 \mathrm{cA}$ & $1873 \mathrm{eA}$ \\
\hline 63 & 2237 bA & $2193 \mathrm{cA}$ & 63 & $2392 \mathrm{cA}$ & $1067 \mathrm{gB}$ \\
\hline 64 & $1761 \mathrm{cA}$ & $1763 \mathrm{dA}$ & 64 & $1700 \mathrm{eB}$ & $2404 \mathrm{dA}$ \\
\hline 65 & 2095 bA & $1980 \mathrm{cA}$ & 65 & $1804 \mathrm{dA}$ & $1906 \mathrm{eA}$ \\
\hline 66 & 2299 bA & $2809 \mathrm{bA}$ & 66 & $1590 \mathrm{eA}$ & $1686 \mathrm{fA}$ \\
\hline 67 & $1563 \mathrm{~dB}$ & $2305 \mathrm{cA}$ & 67 & $1488 \mathrm{eA}$ & $1219 \mathrm{gA}$ \\
\hline 68 & $1658 \mathrm{cB}$ & $3227 \mathrm{aA}$ & 68 & $2348 \mathrm{cA}$ & $1751 \mathrm{fB}$ \\
\hline 69 & $1461 \mathrm{dA}$ & $1927 \mathrm{cA}$ & 69 & $1794 \mathrm{dA}$ & $1711 \mathrm{fA}$ \\
\hline 70 & $1337 \mathrm{~dB}$ & $2824 \mathrm{bA}$ & 70 & $2646 \mathrm{cA}$ & $2237 \mathrm{eA}$ \\
\hline 71 & $2158 \mathrm{bB}$ & $2808 \mathrm{bA}$ & 71 & $1564 \mathrm{eB}$ & $2489 \mathrm{dA}$ \\
\hline 72 & $1149 \mathrm{~dB}$ & $1776 \mathrm{dA}$ & 72 & $2618 \mathrm{cA}$ & $2126 \mathrm{eA}$ \\
\hline 73 & $2433 \mathrm{bA}$ & $1881 \mathrm{cA}$ & 73 & $2424 \mathrm{cA}$ & $1054 \mathrm{gB}$ \\
\hline 74 & $1338 \mathrm{dA}$ & $1632 \mathrm{dA}$ & 74 & $2732 \mathrm{cA}$ & $2379 \mathrm{dA}$ \\
\hline 75 & $2507 \mathrm{bA}$ & $1963 \mathrm{cA}$ & 75 & $3150 \mathrm{bA}$ & $1968 \mathrm{eB}$ \\
\hline 76 & 2229 bA & $2207 \mathrm{cA}$ & 76 & $1332 \mathrm{eB}$ & $2275 \mathrm{eA}$ \\
\hline 77 & $2512 \mathrm{bA}$ & $1618 \mathrm{~dB}$ & 77 & $2484 \mathrm{cA}$ & $2085 \mathrm{eA}$ \\
\hline 78 & $3197 \mathrm{aA}$ & $1261 \mathrm{~dB}$ & 78 & $2292 \mathrm{cA}$ & $2057 \mathrm{eA}$ \\
\hline 79 & $1224 \mathrm{~dB}$ & 2042 cA & 79 & 3078 bA & $3173 \mathrm{cA}$ \\
\hline 80 & 2484 bA & $1479 \mathrm{~dB}$ & 80 & $1340 \mathrm{eA}$ & $1633 \mathrm{fA}$ \\
\hline 81 & $2107 \mathrm{bA}$ & $906 \mathrm{~dB}$ & 81 & $1950 \mathrm{~dB}$ & $4453 \mathrm{aA}$ \\
\hline 82 & $2830 \mathrm{aA}$ & $1494 \mathrm{~dB}$ & 82 & $1608 \mathrm{eB}$ & $2436 \mathrm{dA}$ \\
\hline 83 & $1231 \mathrm{~dB}$ & $1985 \mathrm{cA}$ & 83 & 3480 bA & $2212 \mathrm{eB}$ \\
\hline 84 & $1792 \mathrm{cA}$ & $2244 \mathrm{cA}$ & 84 & $1620 \mathrm{eA}$ & $1843 \mathrm{eA}$ \\
\hline 85 & $1478 \mathrm{dA}$ & $1473 \mathrm{dA}$ & 85 & $1968 \mathrm{~dB}$ & $2558 \mathrm{dA}$ \\
\hline 86 & $3200 \mathrm{aA}$ & $1582 \mathrm{~dB}$ & 86 & $2642 \mathrm{cA}$ & $2397 \mathrm{dA}$ \\
\hline 87 & $1253 \mathrm{dA}$ & $1162 \mathrm{dA}$ & 87 & $1668 \mathrm{eB}$ & $2301 \mathrm{eA}$ \\
\hline 88 & $1709 \mathrm{cA}$ & 1997 cA & 88 & $1152 \mathrm{eA}$ & $1480 \mathrm{fA}$ \\
\hline 89 & $2729 \mathrm{aA}$ & $1619 \mathrm{~dB}$ & 89 & $2172 \mathrm{dA}$ & $1614 \mathrm{fB}$ \\
\hline 90 & $1335 \mathrm{~dB}$ & $2338 \mathrm{cA}$ & 90 & $1926 \mathrm{dA}$ & $1660 \mathrm{fA}$ \\
\hline Avera & 2121 & 2296 & Averag & 2231 & 2358 \\
\hline
\end{tabular}

1: Averages followed by the same lowercase letter in the same column and capital letters in the line (in each crop), do not differ significantly at 5\% probability by the Scott-Knott test.

Rev. Ceres, Viçosa, v. 67, n.1, p. 042-051, jan/feb, 2020 
Table 4: Analysis of joint variance between progenies and crops, for each locality, with respect to the characteristic grain yield $(\mathrm{kg}$ ha-1)

\begin{tabular}{|c|c|c|c|}
\hline \multirow{2}{*}{ Source of variation } & \multirow{2}{*}{$\mathbf{G} \mathbf{L}^{(1)}$} & \multicolumn{2}{|c|}{$\mathbf{Q M}^{(2)}$} \\
\hline & & São Manuel & Araçatuba \\
\hline Bloco/Crop & 4 & 2296415.82 & 360352.07 \\
\hline Crop & 1 & $1653028.02 \mathrm{~ns}$ & $518754.02 \mathrm{~ns}$ \\
\hline Progeny & 89 & $1289843.20 * *$ & $1187038.89 \mathrm{~ns}$ \\
\hline Free pollination & 29 & $1861564.31 * *$ & $1130569.31 * *$ \\
\hline Cross pollination & 29 & $917613.48 * *$ & $844791.89^{* * *}$ \\
\hline Self-pollination & 29 & $957129.58 * *$ & $676509.46^{* * *}$ \\
\hline Between Progeny & 2 & $3221565.69 * *$ & $14371105.81 * *$ \\
\hline Crop x Progeny & 89 & $642306.05^{* *}$ & $1408627.90^{* *}$ \\
\hline Treatment & 179 & & \\
\hline Residue & 356 & 120881.67 & 116764.36 \\
\hline Total & 539 & & \\
\hline$\overline{\mathrm{CV}^{(3)}}$ & & 15.95 & 14.61 \\
\hline
\end{tabular}

Table 5: Average grain yield $\left(\mathrm{kg} \mathrm{ha}^{-1}\right)$ of progenies and crops, for each locality

\begin{tabular}{|c|c|c|c|c|c|}
\hline \multirow{3}{*}{ Progeny } & \multirow{2}{*}{\multicolumn{2}{|c|}{$\begin{array}{c}\text { São Manuel } \\
\text { Crop }\end{array}$}} & \multirow{3}{*}{ Progeny } & \multirow{2}{*}{\multicolumn{2}{|c|}{$\begin{array}{c}\text { Araçatuba } \\
\text { Crop }\end{array}$}} \\
\hline & & & & & \\
\hline & $2004 / 2005$ & $2005 / 2006$ & & $2004 / 2005$ & $2005 / 2006$ \\
\hline 1 & $2887 \mathrm{aA}^{(1)}$ & 2946 bA & 1 & 2494 bA & $2686 \mathrm{dA}$ \\
\hline 2 & $1867 \mathrm{cA}$ & $1860 \mathrm{dA}$ & 2 & $1463 \mathrm{~dB}$ & $2412 \mathrm{dA}$ \\
\hline 3 & $2821 \mathrm{aA}$ & $2772 \mathrm{cA}$ & 3 & $1772 \mathrm{~dB}$ & $2662 \mathrm{dA}$ \\
\hline 4 & $1990 \mathrm{cA}$ & $2004 \mathrm{dA}$ & 4 & $1503 \mathrm{~dB}$ & $3096 \mathrm{dA}$ \\
\hline 5 & $3002 \mathrm{aA}$ & 2988 bA & 5 & $2055 \mathrm{cA}$ & $2455 \mathrm{dA}$ \\
\hline 6 & $2240 \mathrm{bA}$ & $2208 \mathrm{dA}$ & 6 & $2805 \mathrm{bA}$ & $2171 \mathrm{eB}$ \\
\hline 7 & $2887 \mathrm{aA}$ & 2928 bA & 7 & $1584 \mathrm{~dB}$ & $2848 \mathrm{dA}$ \\
\hline 8 & $3282 \mathrm{aA}$ & 3276 bA & 8 & $3904 \mathrm{aA}$ & $2255 \mathrm{eB}$ \\
\hline 9 & $1709 \mathrm{cA}$ & $1674 \mathrm{eA}$ & 9 & $2315 \mathrm{cB}$ & $3524 \mathrm{bA}$ \\
\hline 10 & $2750 \mathrm{aA}$ & $2796 \mathrm{cA}$ & 10 & $1641 \mathrm{~dB}$ & $2881 \mathrm{dA}$ \\
\hline 11 & $2048 \mathrm{cA}$ & $2028 \mathrm{dA}$ & 11 & $2614 \mathrm{bB}$ & $3205 \mathrm{cA}$ \\
\hline 12 & $1960 \mathrm{cA}$ & $1890 \mathrm{dA}$ & 12 & $2032 \mathrm{cB}$ & $2738 \mathrm{dA}$ \\
\hline 13 & $1255 \mathrm{dA}$ & 1086 eA & 13 & $2560 \mathrm{bA}$ & $2487 \mathrm{dA}$ \\
\hline 14 & $1431 \mathrm{dA}$ & $1476 \mathrm{eA}$ & 14 & $1037 \mathrm{~dB}$ & $3064 \mathrm{dA}$ \\
\hline 15 & $2367 \mathrm{bA}$ & $2364 \mathrm{cA}$ & 15 & $2870 \mathrm{bA}$ & $2535 \mathrm{dA}$ \\
\hline 16 & $1591 \mathrm{cA}$ & $1500 \mathrm{eA}$ & 16 & $3122 \mathrm{aA}$ & $2930 \mathrm{dA}$ \\
\hline 17 & $1454 \mathrm{dA}$ & $1494 \mathrm{eA}$ & 17 & $1930 \mathrm{cB}$ & $2685 \mathrm{dA}$ \\
\hline 18 & $2144 \mathrm{bA}$ & $2130 \mathrm{dA}$ & 18 & $2247 \mathrm{cB}$ & $2982 \mathrm{dA}$ \\
\hline 19 & 2189 bA & $2190 \mathrm{dA}$ & 19 & $2778 \mathrm{bA}$ & $2622 \mathrm{dA}$ \\
\hline 20 & $1914 \mathrm{cA}$ & $1884 \mathrm{dA}$ & 20 & $1258 \mathrm{~dB}$ & $2103 \mathrm{eA}$ \\
\hline 21 & $2513 \mathrm{bA}$ & $2484 \mathrm{cA}$ & 21 & $2741 \mathrm{bA}$ & $3280 \mathrm{cA}$ \\
\hline 22 & $2976 \mathrm{aA}$ & 2958 bA & 22 & $1359 \mathrm{dA}$ & $1132 \mathrm{gA}$ \\
\hline 23 & $1597 \mathrm{cA}$ & $1650 \mathrm{eA}$ & 23 & $2635 \mathrm{bA}$ & $1645 \mathrm{fB}$ \\
\hline 24 & $2491 \mathrm{bA}$ & $2574 \mathrm{cA}$ & 24 & $2562 \mathrm{bA}$ & $1885 \mathrm{eB}$ \\
\hline 25 & $2228 \mathrm{bA}$ & $2136 \mathrm{dA}$ & 25 & $1762 \mathrm{dA}$ & $1506 \mathrm{fA}$ \\
\hline 26 & $2497 \mathrm{bA}$ & $2520 \mathrm{cA}$ & 26 & $1911 \mathrm{cA}$ & $2187 \mathrm{eA}$ \\
\hline 27 & $2372 \mathrm{bA}$ & $2352 \mathrm{cA}$ & 27 & $2479 \mathrm{bA}$ & $2213 \mathrm{eA}$ \\
\hline 28 & $2332 \mathrm{bA}$ & $2184 \mathrm{dA}$ & 28 & 3478 aA & $1666 \mathrm{fB}$ \\
\hline 29 & $1421 \mathrm{dA}$ & $1524 \mathrm{eA}$ & 29 & $2123 \mathrm{cA}$ & $1536 \mathrm{fB}$ \\
\hline 30 & $1462 \mathrm{dA}$ & $1488 \mathrm{eA}$ & 30 & $3066 \mathrm{aA}$ & $1440 \mathrm{fB}$ \\
\hline 31 & 2796 aA & $2167 \mathrm{~dB}$ & 31 & $2669 \mathrm{bA}$ & $3052 \mathrm{dA}$ \\
\hline 32 & $1874 \mathrm{cA}$ & $2305 \mathrm{cA}$ & 32 & $3158 \mathrm{aB}$ & 3862 bA \\
\hline 33 & $2832 \mathrm{aA}$ & $2046 \mathrm{~dB}$ & 33 & $2004 \mathrm{cA}$ & $2341 \mathrm{dA}$ \\
\hline
\end{tabular}


Jackson da Silva et al.

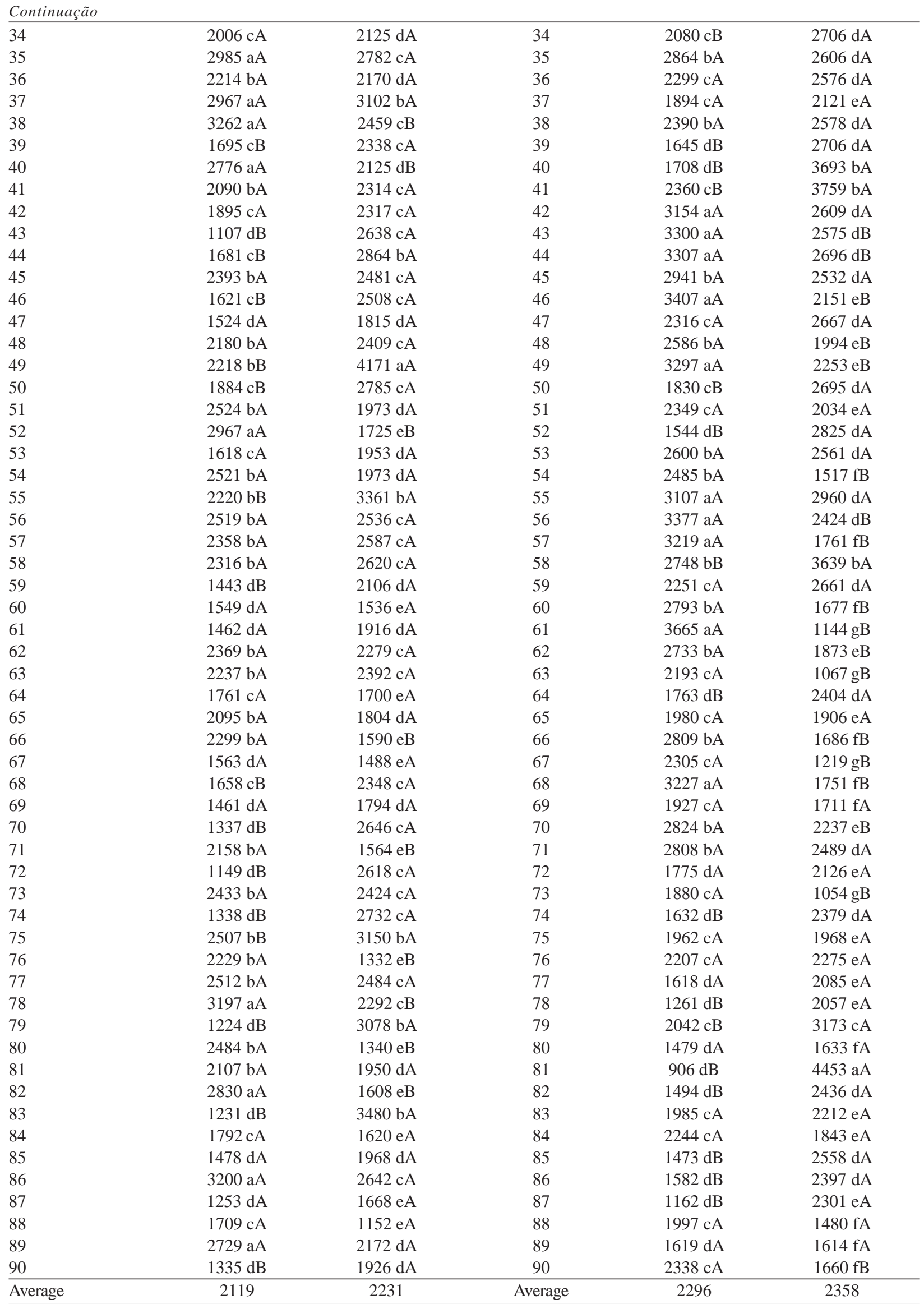

1: Averages followed by the same lowercase letter in the same column and capital letters in the line (in each crop), do not differ significantly at $5 \%$ probability by the Scott-Knott test.

Rev. Ceres, Viçosa, v. 67, n.1, p. 042-051, jan/feb, 2020 
also explained by the good combining ability between its parents, in which each parent had favorable complementary genes with the other parent (Bernini et al., 2013).

With regard to progeny 81 , because it is a progeny originated by self-pollination, which provides the highest level of homozygousness, their grain yield is somewhat surprising, since its productivity was about six times the national average of the 2005/2006 crop (714 kg ha-1, CONAB, 2017), however as castor bean is a kind of reproduction type taken as mixed, may have decreased the deleterious effects deriving from the increase of homozygosity.

In this crop, several of the progenies presented high grain yield in São Manuel were: 1, 5, 7, 8, 22, 37, 75, 79 and 83 showing variation from 2928 to $3480 \mathrm{~kg} \mathrm{ha}^{-1}$, and in Araçatuba 9, 11, 21, 40, 41 and 79, presenting variation from 3173 to $3759 \mathrm{~kg} \mathrm{ha}^{-1}$.

Based on the joint analysis of variance between progenies and crops, for each locality (Table 4), it is possible to notice for the locality of São Manuel that only the vintages factor was not significant, presenting all the other factors significance at $1 \%$ probability by the $\mathrm{F}$ test. With regard to the locality of Araçatuba, the factors crops and progenies were not significant, however, when pollination types are observed and the interaction crop $\mathrm{x}$ progeny is sharp the significance at $1 \%$ probability by the test F. Due to the significance of the crop $\mathrm{x}$ progeny interaction in the two localities the mean comparison test was applied.

Concerning the grain yield of progenies and crops, for each locality (Table 5), it is observed that the locality of Araçatuba provided the highest average productivity, presenting 19 progenies with productivity above $3000 \mathrm{~kg}$ $\mathrm{ha}^{-1}$, which can be attributed to more favorable environmental conditions. However, there were progenies that exhibited high yields in São Manuel presenting 10 progenies with productivity above $3000 \mathrm{~kg} \mathrm{ha}^{-1}$, due to the adaptive capacity of each progeny.

In the analysis of the crop factor, it is noted that in general that the 2005/2006 crop obtained the highest general averages, in the order of 2231 and $2358 \mathrm{~kg} \mathrm{ha}^{-1}$ for São Manuel and Araçatuba, respectively, indicating that in this crop there may have been better conditions for expression of progenies productive potential.

The best combinations in San Manuel, average between crops, were progenies $1,5,7,8,22,37,49$ and 86, ranging from 2907 to $3279 \mathrm{~kg} \mathrm{ha}^{-1}$, noting that progenies 37 and 49 were the most productive. For the locality of Araçatuba, the best average values between the crops, were derived from the combination of progenies $8,9,11,16,21,32,41$, $43,44,49,55,56$ and 58, these progenies having yields varying from 2900 to $3510 \mathrm{~kg} \mathrm{ha}^{-1}$, and the progeny 81 with crop 2005/2006 with yield of $4453 \mathrm{~kg} \mathrm{ha}^{-1}$.

It should be noted that the best progenies in São Manuel not repeated from one crop to another, as well as for the locality of Araçatuba, indicating that the vast majority of progenies exhibit low productivity stability (Ribeiro et al., 2008).

However, productivity stability is an important element taken into account in the choice of cultivar by the producer, in this way it can be noticed that the progenies as intermediate productivities, within the sampled group, were the most stable, and it is emphasized that their productivities are excellent when compared to the regional average of $443 \mathrm{~kg} \mathrm{ha}^{-1}$ in the 2016/2017 crop (CONAB, 2017).

Progenies 1, 5, 6, 8, 15, 18, 19, 21, 27, 31, 35, 36, 38, 45, $49,55,56$, and 58 exhibit a certain level of stability with productivity at over $2100 \mathrm{~kg} \mathrm{ha}^{-1}$, in different cities and crops, being selected by these aspects, and can be used to obtain new cultivars and hybrids (Machado et al., 2008).

Table 6: Summary of variance analyzes of the three types of pollination for castor bean progenies in two localities in the State of São Paulo and in two crops

\begin{tabular}{|c|c|c|c|c|c|c|}
\hline Crops & Localities & Progenies & $\mathbf{Q M P}^{(1)}$ & $\mathbf{Q M E}^{(2)}$ & Average & $\mathbf{C V}^{(3)}$ \\
\hline \multirow{6}{*}{$2004 / 2005$} & \multirow{3}{*}{ São Manuel } & Free $P$. & $918868.97 * *$ & \multirow{3}{*}{107545.93} & 2189.36 & \multirow{3}{*}{15.46} \\
\hline & & Cross $\mathrm{P}$. & $888429.87 * *$ & & 2201.14 & \\
\hline & & Self P. & $1089343.40 * *$ & & 1971.23 & \\
\hline & \multirow{3}{*}{ Araçatuba } & Free P. & $1395197.52 * *$ & \multirow{3}{*}{139406.58} & 2270.01 & \multirow{3}{*}{16.25} \\
\hline & & Cross $\mathrm{P}$. & $956515.92 * *$ & & 2589.35 & \\
\hline & & Self P. & $1148519.56^{* *}$ & & 2029.68 & \\
\hline \multirow{6}{*}{$2005 / 2006$} & \multirow{4}{*}{ São Manuel } & Free P. & $948342.28 * *$ & \multirow{4}{*}{134217.39} & 2178.80 & \multirow{4}{*}{16.41} \\
\hline & & Cross $\mathrm{P}$. & $811487.90 * *$ & & 2409.68 & \\
\hline & & Self P. & $1012533.89 * *$ & & 2105.22 & \\
\hline & & Free P. & $1112362.50^{* *}$ & & 2427.68 & \\
\hline & \multirow{2}{*}{ Araçatuba } & Cross $\mathrm{P}$. & $1004970.94 * *$ & \multirow[t]{2}{*}{94122.13} & 2607.72 & \multirow[t]{2}{*}{13.00} \\
\hline & & Self $\mathrm{P}$. & $1335967.10^{* *}$ & & 2039.61 & \\
\hline
\end{tabular}

\footnotetext{
** Significant at $1 \%$ probability by $\mathrm{F}$ test. 1: Mean square of progenies; 2: Mean square of the residue; 3: Coefficient of variation.
} 
Jackson da Silva et al.

Table 7: Estimates of genetic parameters with relation to the three types of pollination for castor bean progenies in two localities in the State of São Paulo and in two crops

\begin{tabular}{|c|c|c|c|c|c|c|c|}
\hline Crops & Localities & Progenies & $\sigma^{2} p^{(1)}$ & $\sigma^{2} \mathbf{e}^{(2)}$ & $h^{2} m^{(3)}$ & $\Delta \mathbf{G}^{(4)}$ & G \% ${ }^{(5)}$ \\
\hline \multirow{6}{*}{$2004 / 2005$} & \multirow{3}{*}{ São Manuel } & Free $\mathrm{P}$. & $270441.01 * *$ & 107545.94 & 0.88 & 684.12 & 31.25 \\
\hline & & Cross P. & $260294.64 * *$ & 107545.94 & 0.88 & 669.64 & 30.42 \\
\hline & & Self P. & $327265.82 * *$ & 107545.94 & 0.90 & 760.34 & 38.57 \\
\hline & \multirow{3}{*}{ Araçatuba } & Free $\mathrm{P}$. & $418596.98 * *$ & 139406.58 & 0.90 & 859.34 & 37.86 \\
\hline & & Cross P. & $272369.78 * *$ & 139406.58 & 0.85 & 675.31 & 26.08 \\
\hline & & Self P. & $336370.99 * *$ & 139406.58 & 0.88 & 761.09 & 37.50 \\
\hline \multirow{6}{*}{ 2005/2006 } & \multirow{3}{*}{ São Manuel } & Free $\mathrm{P}$. & $271374.96 * *$ & 134217.39 & 0.86 & 675.73 & 31.01 \\
\hline & & Cross P. & $225756.83 * *$ & 134217.39 & 0.83 & 607.70 & 25.22 \\
\hline & & Self P. & $292772.16^{* *}$ & 134217.39 & 0.87 & 705.53 & 33.51 \\
\hline & \multirow{3}{*}{ Araçatuba } & Free $P$. & $339413.45^{* *}$ & 94122.13 & 0.92 & 780.36 & 32.14 \\
\hline & & Cross P. & $303616.27 * *$ & 94122.13 & 0.91 & 734.41 & 28.16 \\
\hline & & Self P. & $413948.32 * *$ & 94122.13 & 0.93 & 868.43 & 42.58 \\
\hline
\end{tabular}

** Significant at $1 \%$ probability by $\mathrm{F}$ test. 1: Estimates of genetic variance between progenies $\left(\mathrm{kg} \mathrm{h}^{-1}\right)^{2}$. 2: Estimates of environmental variance $\left(\mathrm{kg} \mathrm{ha}^{-1}\right)^{2}$. 3: Estimates of the coefficient of heritability at the level of progeny average. 4: Estimated genetic progress with $20 \%$ of selection intensity $\left(\mathrm{kg} \mathrm{ha}^{-1}\right)$. 5: Genetic progress estimated in relation to the average of each pollination type for the characteristic grain yield.

It can be observed that of the more stable preselected progenies, 9 are from free pollination and the other 9 from cross-pollination, can these 9 progenies of crosspollination resulting in highly productive hybrids (Bernini et al., 2013).

There was variation in the means of pollination types both for the 2004/2005 and 2005/06 crop how much for the cities of São Manuel and Araçatuba at the 1\% probability level by the $\mathrm{F}$ test, indicating the possibility of success with the selection of the best genotypes (Table 6).

The estimated coefficients of heritability at the level of progeny averages in relation to grain yield character varied from 0.83 to 0.93 , being observed the highest values in the 2005/2006 crop in the city of Araçatuba (Table 7), it is noteworthy that in the same condition, it is also noticeable the highest genetic gains estimated with the selection of progenies $20 \%$ better, reaching a gain of $42.58 \%$ in relation to the means of the progenies that were self-pollinated.

The significance of the variances of progenies (genetic) at the $1 \%$ probability level by the $\mathrm{F}$ test, for the four experiments, indicates the existence of genetic variability between the progenies of each type of pollination and allows for genetic gains through selection (Silveira et al., 2016). This significant effect, in particular the progenies from self-pollination, in which of the four experiments obtained greater percentage gains in three of them, may be related to the higher level of homozygosis, expressing with it greater divergence between the progenies, the one that allows the percentage of larger genetic gains (Vargas-Reeve et al., 2013).

Its valid mention that, generally, in the city of Araçatuba were observed the highest estimates of heritability, indicating that besides the possibility of genetic gain with the progeny selection, this environment also favors the choice of more stable progenies, being this reasoning shared by Silva et al. (2011), that observed relation between heritability and phenotypic stability face of environmental variations (Silveira et al., 2016; Passos et $a l ., 2010)$. High estimates of heritability also demonstrate good genetic control, assuming that the most of the observed variation in castor bean plants was genetic in nature (Cruz Neto et al., 2016).

To the what it concerns estimated genetic gains with selection intensity of $20 \%(\Delta \mathrm{G})$ and the estimated genetic gains in percentage of the average of each type of pollination (G\%), it can be stated that they presented the highest values for the locality of Araçatuba, being this the local more suitable for selection, because the progenies selected for this environment may have greater stability.

It is noteworthy, which at the time in which the experiments were conducted, the region of the two cities is characterized by higher rainfall and higher temperatures, these conditions, appropriate for the good development of the castor bean crop, the what may have influenced the progenies to express their genotypic potential, which reflected in high estimates of heritability, allowing greater genetic gains with the selection (Cruz Neto et al., 2016).

\section{CONCLUSIONS}

Both the locality of Araçatuba and the 2005/2006 crop provided better expressions of the productive potential of the progenies, indicating their genetic potential, which facilitates and increases the precision of the selection of the best genotypes. 
The progenies selected were progenies $1,5,6,8,15,18$, 19, 21 and 27 (caused by free pollination), as well as progenies $31,35,36,38,45,49,55,56$ and 58 (resulting from cross-pollination) all present productivity above $2100 \mathrm{~kg}$ $\mathrm{ha}^{-1}$, regardless of the crop and the city, suggesting that there was some level of stability, and can they be used to obtain new cultivars and hybrids.

The locality of Araçatuba presented the highest estimates of heritability, especially progenies self pollinated, in which they provided that lines presented more stable productive behavior, which increases the efficiency of the selection of superior genotypes.

Genotype interaction with the location indicated productive materials and adapted to the state of São Paulo, showing that there are managements that can solve the productivity problem, and consequently, increase the production of this crop.

\section{ACKNOWLEDGEMENTS, FINANCIAL SUPPORT AND FULL DISCLOSURE}

To the National Council of Scientific and Technological Development Brazil (CNPq), for the financial assistance (process n ${ }^{\circ} 310794 / 2015-3$ ) and (process no 134541 /2016-2).

There is no conflict of interests in the conduction and publication of this article.

\section{REFERENCES}

Alvares CA, Stape JL, Sentelhas PC, Gonçalves JLM \& Sparovek G (2014) Köppen's climate classification map for Brazil. Meteorologische Zeitschrift, 22:711-728.

Barbosa JC \& Maldonado Junior W (2015) AgroEstat - sistema para análises estatísticas de ensaios agronômicos. Jaboticabal, FCAV/UNESP. 396p

Bernini CS, Paterniani MEAGZ, Duarte AP, Gallo PB, Guimarães OS \& Rovaris SRS (2013) Depressão endogâmica e heterose de híbridos de populações $\mathrm{F}_{2}$ de milho no estado de São Paulo. Bragantia, 72:217-223.

CONAB - Companhia Nacional de Abastecimento (2017) Safras: série histórica das safras. Available at: <https://www.conab.gov.br/ info-agro/safras/serie-historica-das-safras?start=10>. Accessed on: May 20 2018.

Cruz Neto AJ, Rosa RCC, Oliveira EJ, Sampaio SR, Santos IS, Souza PU, Passos AR \& Jesus ON (2016) Genetic parameters, adaptability and stability to selection of yellow passion fruit hybrids. Crop Breeding and Applied Biotechnology, 16:321-329.

Cruz AD, Regazzi AJ \& Carneiro PCS (2012) Modelos Biométricos Aplicados ao Melhoramento Genético. Viçosa, Editora UFV. $514 p$.

Daronch DJ, Peluzio JM, Afferri FS, Tavares AT \& Souza CM (2019) Eficiência ambiental e divergência genética de genótipos de soja na região central do Tocantins. Cultura Agronômica, 28:01-18

FAO - Food and Agriculture Organization of the United Nations (2016) Faostat - Countries by commodity. Available at: <http:/ /www.fao.org/faostat/en/\#rankings/countries_by_commodity >. Accessed on: May 20 2018
Ferreira PV (2006) Melhoramento de plantas: métodos de melhoramento. Maceió, Edufal. 110p.

Gaya LG, Mourão GB \& Ferraz JBS (2006) Aspectos genéticoquantitativos de características de desempenho, carcaça e composição corporal em frangos. Ciência Rural, 36:709-716.

Goneli ALD, Corrêa PC, Oliveira APLR, Hartmann Filho CP \& Oba GC (2018) Castor beans quality subjected to different storage temperatures and periods. Engenharia Agrícola, 38:361-368.

Machado JC, Souza JC, Ramalho MAP \& Lima JL (2008) Estabilidade de produção de híbridos simples e duplos de milho oriundos de um mesmo conjunto gênico. Bragantia, 67:627-631.

Passos AR, Silva SA, Souza CS, Souza CMM \& Fernandes LS (2010) Parâmetros genéticos de caracteres agronômicos em genótipos de mamoneira. Pesquisa Agropecuária Brasileira, 45:709-714.

Ribeiro ND, Antunes IF, Souza JF \& Poersch NL (2008) Adaptação e estabilidade de produção de cultivares e linhagens-elite de feijão no Estado do Rio Grande do Sul. Ciência Rural, 38:24342440 .

Silva JAG, Bandeira TP, Manjabosco CD, Krüguer CAMB, Silva SDA, Crestani M \& Carbonera R (2011) Caracterização e herdabilidade em caracteres morfológicos e fisiológicos da mamona. Revista Brasileira Agrociência, 17:348-358.

Silveira LCI, Brasileiro BP, Kist V, Weber H, Daros E, Peternelli LA \& Barbosa MHP (2016) Selection in energy cane families. Crop Breeding and Applied Biotechnology, 16:298-306.

Torres FE, Teodoro PE, Sagrilo E, Ceccon G \& Correa AM (2015) Interação genótipo $\mathrm{x}$ ambiente em genótipos de feijão-caupi semiprostrado via modelos mistos. Bragantia, 74:15-20.

Vargas-Reeve F, Mora F, Perret S \& Scapim CA (2013) Heritability of stem straightness and genetic correlations in Eucalyptus cladocalyx in the semi-arid region of Chile. Crop Breeding and Applied Biotechnology, 13:107-112.

Vencovsky R (1980) Herança quantitativa. In Paterniani E (Ed.) Melhoramento e produção de milho no Brasil. Piracicaba, Fundação Cargill. p.122-201. 